\title{
Integrality properties in the Moduli Space of Elliptic Curves: CM Case
}

\author{
Stefan Schmid
}

\begin{abstract}
A result of Habegger Hab15 shows that there are only finitely many singular moduli such that $j$ or $j-\alpha$ is an algebraic unit. The result uses Duke's Equidistribution Theorem and is thus not effective. For a fixed $j$-invariant $\alpha \in \overline{\mathbb{Q}}$ of an elliptic curve without complex multiplication, we prove that there are only finitely many singular moduli $j$ such that $j-\alpha$ is an algebraic unit. The difference to Hab15] is that we give explicit bounds.
\end{abstract}

\section{Introduction}

We denote Klein's modular function defined on the upper half-plane by $j$. A singular modulus is the value of the $j$-function evaluated at an imaginary quadratic $\tau$. Singular moduli correspond to the $j$-invariants of elliptic curves with complex multiplication. A classical result by Kronecker states that singular moduli are algebraic integers.

In 2011, Masser asked at the AIM workshop on unlikely intersections in algebraic groups and Shimura varieties in Pisa, if there are only finitely many singular moduli that are algebraic units. His question was motivated by BMZ13 and a result on the André-Oort conjecture. In 2014, Habegger gave an answer in [Hab15] to this question by proving that at most finitely many singular moduli are algebraic units. No examples were known at that time. He also proved that only finitely many singular moduli exist such that $j-\alpha$ is a unit. Here examples are known, e.g. one can take $\alpha=1$ and then $j=0$ is a solution. His results in Hab15 rely on Duke's Equidistribution Theorem and are thus not effective. In 2018 Bilu, Habegger and Kühne proved in BHK18 that no singular modulus is a unit.

In the work at hand we want to give effective bounds for the number of singular moduli $j$ such that $j-\alpha$ is a unit, where $\alpha \in \overline{\mathbb{Q}}$ is the $j$-invariant of an elliptic curve without complex multiplication. More specifically, we obtain a bound on the discriminant of the endomorphism ring of the singular moduli satisfying the condition. See a recent result of Li [Li18] for differences of singular moduli.

We fix a singular modulus $j$. Elliptic curves with $j$-invariant $j$ have the same full endomorphism ring. Let $\Delta$ denote the discriminant of this ring. We want to prove the following result. 
Theorem 1.1 Let $j$ be a singular modulus and let $\Delta$ be its discriminant. Let $\alpha$ be an algebraic number that is the $j$-invariant of an elliptic curve without complex multiplication. If we assume that $j-\alpha$ is an algebraic unit, then $|\Delta|$ is bounded from above by an explicit constant. Thus there are only finitely many singular moduli $j$ such that $j-\alpha$ is an algebraic unit. The constant can be found on page 23.

The sketch of the proof is as follows. Write $j(\xi)=\alpha \in \overline{\mathbb{Q}}$ where the elliptic curve associated to $\alpha$ does not have complex multiplication. We can assume that $\xi$ is in the classical fundamental domain $\mathcal{F}$ of the upper half-plane. To a singular modulus $j$ we have attached an elliptic curve with complex multiplication. We can write $\Delta=D f^{2}$ where $f$ is the conductor of the endomorphism ring in the full ring of integers of $\mathbb{Q}(\sqrt{\Delta})$, and $D$ is the discrimiant of that field. The Galois conjugates of $j$ form a full orbit of length the class number $\mathcal{C}(\Delta)$. We write $\mathcal{C}(\Delta ; \xi ; \varepsilon)$ for the number of singular moduli with discriminant $\Delta$ that can be written in the form $j(\tau)$ with $\tau \in \mathcal{F}$ and such that $|\tau-\xi|<\varepsilon$. We prove an explicit bound on $\mathcal{C}(\Delta ; \xi ; \varepsilon)$ which is given by

$$
\mathcal{C}(\Delta ; \xi ; \varepsilon) \leq F(\Delta)\left(32|\Delta|^{1 / 2} \varepsilon^{2} \log \log \left(|\Delta|^{1 / 2}\right)+11|\Delta|^{1 / 2} \varepsilon+2\right)
$$

for $|\Delta| \geq 10^{14}$ and $0<\varepsilon<1 / 2$. Here

$$
F(\Delta)=\max \left\{2^{\omega(a)} ; a \leq|\Delta|^{1 / 2}\right\}
$$

and $\omega(n)$ is the number of distinct prime divisors of $n$.

The idea is to give lower and upper bounds of the logarithmic (Weil) height of $j-\alpha$ that contradict each other. The height of an algebraic number basically measures its complexity. Let $K$ be a number field containing $\beta \in \overline{\mathbb{Q}}$. If $M_{K}$ is a set of representatives of non-trivial absolute values extending the $p$-adic absolute values and the usual absolute value, and $\left[K_{\nu}: \mathbb{Q}_{\nu}\right], \nu \in M_{K}$, denotes the local degrees, then the height of $\beta \in \overline{\mathbb{Q}}$ is defined by

$$
h(\beta)=\frac{1}{[K: \mathbb{Q}]} \sum_{\nu \in M_{K}}\left[K_{\nu}: \mathbb{Q}_{\nu}\right] \log \max \left\{1,|\beta|_{\nu}\right\} .
$$

The definition does not depend on the number field $K$. If $\beta$ is a unit in the ring of integers, then the height can be computed through

$$
h(\beta)=h\left(\beta^{-1}\right)=\frac{1}{[K: Q]} \sum_{\left|\sigma\left(\beta^{-1}\right)\right|>1} \log \left|\sigma\left(\beta^{-1}\right)\right|=-\frac{1}{[K: Q]} \sum_{|\sigma(\beta)|<1} \log |\sigma(\beta)| .
$$

where $\sigma$ runs over all field embeddings $\sigma: K \hookrightarrow \mathbb{C}$. More information on heights can be found in [BG07].

Now if $j-\alpha$ is an algebraic unit, the height can be bounded as

$$
h(j-\alpha) \ll \frac{\mathcal{C}\left(\Delta ; \xi^{\prime} ; \varepsilon\right)}{\mathcal{C}(\Delta)}(\log |\Delta|)^{4}-\log \varepsilon
$$


for some $\xi^{\prime} \in \mathcal{F}$ associated to $\xi$. The constant in the inequality depends on $\alpha$. We put $E(\Delta)=F(\log |\Delta|)^{4}$ and roughly choose $\varepsilon$ to be

$$
\varepsilon=\frac{\mathcal{C}(\Delta)}{E(\Delta)|\Delta|^{1 / 2}}
$$

If we substitute this and (1) into (2) and use estimates for $\omega(n)$ by Robin Rob83] we get

$$
h(j-\alpha) \ll \frac{E(\Delta)}{\mathcal{C}(\Delta)}+\log \frac{E(\Delta)|\Delta|^{1 / 2}}{\mathcal{C}(\Delta)} .
$$

To bound $|\Delta|$ from above we need lower bounds for the height of $j-\alpha$. One can prove

$$
h(j-\alpha) \gg \log |\Delta|
$$

and

$$
h(j-\alpha) \gg \frac{|\Delta|^{1 / 2}}{\mathcal{C}(\Delta)} .
$$

The first inequality follows from work of Colmez [Col98] and Nakkajima-Taguchi [NT91], and the second inequality is more elementary. Again the bounds depend on $\alpha$. Combining the lower bounds with the upper bound from (3) we obtain

$$
\max \left\{\frac{|\Delta|^{1 / 2}}{\mathcal{C}(\Delta)}, \log |\Delta|\right\} \ll \frac{E(\Delta)}{\mathcal{C}(\Delta)}+\log \frac{|\Delta|^{1 / 2}}{\mathcal{C}(\Delta)}+\log E(\Delta)
$$

for large $|\Delta|$. Further analysis shows that $E(\Delta)|\Delta|^{-1 / 2}=|\Delta|^{o(1)}$ and $\log E(\Delta) / \log |\Delta|=$ $o(1)$. Thus the inequality can not hold for large values of $|\Delta|$. All constants in the above deductions can be made explicit, but some are very large.

\section{Bounding points in the fundamental domain}

Let $\mathcal{Q}(\Delta)$ be the set $\left(\subseteq \mathbb{Z}^{3}\right)$ of coefficients representing reduced primitive, positive definite quadratic forms with discriminant $\Delta$ and let $\mathcal{C}(\Delta)$ be the class number. We will write $\Delta=D f^{2}$ throughout this exposition, where $D$ is the discriminant of the imaginary quadratic field $\mathbb{Q}(\sqrt{\Delta})$ and $f \in \mathbb{N}$ is called the conductor. For $\xi \in \mathcal{F}$ and $\varepsilon>0$ we define

$$
\mathcal{C}(\Delta ; \xi ; \varepsilon)=\#\left\{(a, b, c) \in \mathcal{Q}(\Delta) ;\left|\frac{-b+\Delta^{1 / 2}}{2 a}-\xi\right|<\varepsilon\right\}
$$

We also define the function $F$ of $\Delta$ by

$$
F=F(\Delta)=\max \left\{2^{\omega(a)} ; a \leq|\Delta|^{1 / 2}\right\},
$$

where $\omega(n)$ is the number of distinct prime divisors of $n$. We also define the modified conductor by

$$
\tilde{f}= \begin{cases}f & D \equiv 1 \bmod 4 \\ 2 f & D \equiv 0 \bmod 4 .\end{cases}
$$


Then $\Delta / \tilde{f}^{2}$ is square-free.

Let $\sigma_{k}(n)=\sum_{d \mid n} d^{k}$. We are now ready to state the first lemma that gives a bound on the $\tau$ in a neighborhood of a fixed point such that $j(\tau)$ is a singular modulus of fixed discriminant. While this is a generalization of Theorem 2.1 in [BHK18, the constants are not as good as in that very paper.

Lemma 2.1 Let $\Delta$ be a negative integer, $y=\operatorname{Im}(\xi) \geq \sqrt{3} / 2$ and $0<\varepsilon<1 / 4$. Then

$$
\mathcal{C}(\Delta ; \xi ; \varepsilon) \leq F(\Delta)\left(32 \frac{\sigma_{1}(\tilde{f})}{\tilde{f}} \frac{|\Delta|^{1 / 2}}{4 y^{2}-1} \varepsilon^{2}+8 \sigma_{0}(\tilde{f})\left|\frac{\Delta}{3}\right|^{1 / 4} \varepsilon+8 \frac{|\Delta|^{1 / 2}}{4 y^{2}-1} \varepsilon+2\right)
$$

Proof. We start with $|\tau-\xi|<\varepsilon$. This implies that the real and imaginary parts satisfy

$$
\begin{aligned}
& \operatorname{Im}(\tau) \in(\operatorname{Im}(\xi)-\varepsilon, \operatorname{Im}(\xi)+\varepsilon) \\
& \operatorname{Re}(\tau) \in(\operatorname{Re}(\xi)-\varepsilon, \operatorname{Re}(\xi)+\varepsilon) .
\end{aligned}
$$

Now $\tau$ is of the form $(-b+\sqrt{\Delta}) / 2 a$ and thus $\operatorname{Im}(\tau)=|\Delta|^{1 / 2} / 2 a$ and $\operatorname{Re}(\tau)=-b / 2 a$. This amounts to

or equivalently

$$
y-\varepsilon<\frac{|\Delta|^{1 / 2}}{2 a}<y+\varepsilon
$$

$$
a \in\left(\frac{|\Delta|^{1 / 2}}{2 y+2 \varepsilon}, \frac{|\Delta|^{1 / 2}}{2 y-2 \varepsilon}\right)=: I .
$$

For $b$ we obtain

$$
2 a(\operatorname{Re}(\xi)-\varepsilon)<-b<2 a(\operatorname{Re}(\xi)+\varepsilon),
$$

so $b$ lies in an interval of length $4 a \varepsilon$. For two integers $m$ and $n$ we denote by $\operatorname{gcd}_{2}(m, n)$ the greatest common divisor $d$ of $m$ and $n$ such that $d^{2} \mid m$ and $d^{2} \mid n$. We have $\Delta=b^{2}-4 a c$, so in particular $b^{2} \equiv \Delta \bmod a$. Thus, the residue classes modulo $a / \operatorname{gcd}_{2}(a, \Delta)$ of $b \in \mathbb{Z}$ satisfying $b^{2} \equiv \Delta \bmod a$ is at most $2^{\omega(a / \operatorname{gcd}(a, \Delta))+1}$ by Lemma 2.4 in [BHK18]. Note that we have $\omega(a / \operatorname{gcd}(a, \Delta)) \leq \omega(a)$. But $b$ also lies in the interval given in equation (4), so that by Lemma 2.5 of [BHK18] there are at most

$$
\left(\frac{2 a(\operatorname{Re}(\xi)+\varepsilon)-2 a(\operatorname{Re}(\xi)-\varepsilon)}{a / \operatorname{gcd}_{2}(a, \Delta)}+1\right) 2^{\omega(a)+1}=\left(4 \varepsilon \operatorname{gcd}_{2}(a, \Delta)+1\right) 2^{\omega(a)+1}
$$

possible $b$ 's for any fixed $a$. We have $a \leq|\Delta / 3|^{1 / 2}$ by Lemma 5.3.4 in Coh13, so that $2^{\omega(a)} \leq F$. Using the equality in (5) and applying Lemma 5.3.4 of [Coh13] in the second inequality we get

$$
\begin{aligned}
\mathcal{C}(\Delta ; \xi ; \varepsilon) & \leq 8 \varepsilon \sum_{a \in I \cap \mathbb{Z}} \operatorname{gcd}_{2}(a, \Delta) 2^{\omega(a)}+2 \sum_{a \in I \cap \mathbb{Z}} 2^{\omega(a)} \\
& \leq 8 \varepsilon F \sum_{\substack{a \in I \cap \mathbb{Z}\\
}} \operatorname{gcd}_{2}(a, \Delta)+2 F \#(I \cap \mathbb{Z}) \\
& \leq 8 \varepsilon F \sum_{\substack{d^{2}|\Delta \\
d \leq| \Delta /\left.3\right|^{1 / 4}}} d \cdot \#\left(I \cap d^{2} \mathbb{Z}\right)+2 F \#(I \cap \mathbb{Z}) .
\end{aligned}
$$


Here we used Lemma 5.3.4 in Coh13 in the last step again. But since $\Delta / \tilde{f}^{2}$ is squarefree we obtain

$$
\mathcal{C}(\Delta ; \xi ; \varepsilon) \leq 8 \varepsilon F \sum_{\substack{d|\tilde{f} \\ d \leq| \Delta /\left.3\right|^{1 / 4}}} d\left(\frac{|I|}{d^{2}}+1\right)+2 F(|I|+1),
$$

where $|I|$ is the length of $I$. This can be further simplified to

$$
\begin{aligned}
\mathcal{C}(\Delta ; \xi ; \varepsilon) & \leq 8 \varepsilon F|I| \sum_{\substack{d|\tilde{f} \\
d \leq| \Delta /\left.3\right|^{1 / 4}}} d^{-1}+8 \varepsilon F \sum_{\substack{d|\tilde{f} \\
d \leq| \Delta /\left.3\right|^{1 / 4}}} d+2 F(|I|+1) \\
& \leq 8 \varepsilon F|I| \frac{\sigma_{1}(\tilde{f})}{\tilde{f}}+8 \varepsilon F\left|\frac{\Delta}{3}\right|^{1 / 4} \sigma_{0}(\tilde{f})+2 F(|I|+1) .
\end{aligned}
$$

The length of $I$ can be estimated by

$$
\frac{|\Delta|^{1 / 2}}{2 y-2 \varepsilon}-\frac{|\Delta|^{1 / 2}}{2 y+2 \varepsilon}=|\Delta|^{1 / 2} \frac{2 y+2 \varepsilon-(2 y-2 \varepsilon)}{4 y^{2}-4 \varepsilon^{2}} \leq|\Delta|^{1 / 2} \frac{4 \varepsilon}{4 y^{2}-1} .
$$

This gives the desired inequality.

The next corollary gives a bound on $\mathcal{C}(\Delta ; \xi ; \varepsilon)$ just in terms of $\Delta$ and $\varepsilon$.

Corollary 2.2 For $|\Delta| \geq 10^{14}$ and $0<\varepsilon<1 / 4$ we have

$$
\mathcal{C}(\Delta ; \xi ; \varepsilon) \leq F(\Delta)\left(32|\Delta|^{1 / 2} \varepsilon^{2} \log \log \left(|\Delta|^{1 / 2}\right)+11|\Delta|^{1 / 2} \varepsilon+2\right) .
$$

Proof. For $|\Delta| \geq 10^{14}$ we can find the following results as Lemma 2.8 in BHK18

$$
\begin{gathered}
\sigma_{0}(\tilde{f}) \leq|\Delta|^{0.192} \leq|\Delta|^{1 / 4} \\
\sigma_{1}(\tilde{f}) / \tilde{f} \leq 1.842 \log \log \left(|\Delta|^{1 / 2}\right) .
\end{gathered}
$$

Moreover, we have $y \geq \sqrt{3} / 2$ and thus $4 y^{2}-1 \geq 2$. Hence

$$
\frac{\sigma_{1}(\tilde{f})}{\tilde{f}} \frac{|\Delta|^{1 / 2}}{4 y^{2}-1} \varepsilon^{2} \leq|\Delta|^{1 / 2} \varepsilon^{2} \log \log \left(|\Delta|^{1 / 2}\right)
$$

and

$$
\begin{aligned}
8 \sigma_{0}(\tilde{f})\left|\frac{\Delta}{3}\right|^{1 / 4} \varepsilon+8 \frac{|\Delta|^{1 / 2}}{4 y^{2}-1} \varepsilon & \leq \frac{8}{3^{1 / 4}}|\Delta|^{1 / 2} \varepsilon+4|\Delta|^{1 / 2} \varepsilon \\
& \leq 7|\Delta|^{1 / 2} \varepsilon+4|\Delta|^{1 / 2} \varepsilon
\end{aligned}
$$

which gives the claimed statement. 


\section{Height bounds}

From now on $\alpha$ will be the $j$-invariant of an elliptic curve without complex multiplication. As a preparation we will start with some properties of the $j$-function.

Lemma 3.1 The function $j(1 / 2+i y)$ as a function of $y$ on the interval $[\sqrt{3} / 2, \infty)$ is real and decreasing. The function $j\left(e^{i \theta}\right)$ on the interval $[\pi / 3, \pi / 2]$ is real and increasing, and we have $j\left(e^{i \pi / 2}\right)=j(i)=1728$. The function $j(i y)$ on the interval $[1, \infty)$ is real and increasing.

Proof. Recall $q=e^{2 \pi i \tau}$. For $\tau=\frac{1}{2}+i y$ with $y \geq \sqrt{3} / 2$ we have $q=e^{\pi i} e^{-2 \pi y}=-e^{-2 \pi y}$. Thus $j(\tau)$ is real since all non-zero coefficients of $j$ are positive integers. See for example Leh42 for details. We have $j(1 / 2+i \sqrt{3} / 2)=0$ and from page 227 of Cox11 we know $j(1 / 2+\sqrt{-7} / 2)=-15^{3}$. But the map $y \mapsto j(1 / 2+i y)$ is continuous and injective because $j$ is continuous and injective as a function on $\mathcal{F}$. Thus, it is monotonically decreasing.

Similarly, if $\tau=i y$ with $y \geq 1$, then $q=e^{-2 \pi y}$. We know $j(i)=1728=12^{3}$ and again from page 227 of Cox11] we know $j(i \sqrt{2})=20^{3}$. The same argument as before shows the claim for the map $y \mapsto j(i y)$.

It remains to show that $j\left(e^{i \theta}\right)$ is real because in that case $j\left(e^{i \pi / 3}\right)=0$ and $j\left(e^{i \pi / 2}\right)=$ 1728 imply the monotonicity. Write $\tau=e^{i \theta}$. We have $\bar{q}=e^{2 \pi i(-\bar{\tau})}$ and

$$
\overline{j(\tau)}=(\bar{q})^{-1}+\sum_{n=0}^{\infty} c_{n}(\bar{q})^{n}=j(-\bar{\tau})
$$

But $j$ is $\mathrm{SL}_{2}(\mathbb{Z})$-invariant so that $\overline{j(\tau)}=j(-\bar{\tau})=j(\tau)$ since $|\tau|=1$. Therefore, $j(\tau)$ must be real. This completes the proof.

The next two statements tell us something about the growth of $j(\tau)$ as $|\tau|$ goes to infinity.

Proposition 3.2 If $\tau$ is in $\mathcal{F}$, then ||$j(\tau)\left|-e^{2 \pi \operatorname{Im}(\tau)}\right| \leq 2079$.

This result can be found in Lemma 1 of [BMZ13]. We are going to prove the following result, which is of similar nature.

Lemma 3.3 Let $\tau$ be complex with $\operatorname{Im}(\tau) \geq 1 / 2$. Then

$$
|| j(\tau)\left|-e^{2 \pi \operatorname{Im}(\tau)}\right| \leq 287473
$$

Proof. We have $j(\tau)=q^{-1}+c_{0}+c_{1} q+\cdots$, where as usual $q=e^{2 \pi i \tau}$. Recall that the coefficients of the $q$-expansion of $j$ are all non-negative integers. Then ||$j|-| q^{-1}|| \leq$ $\sum_{n=0}^{\infty} c_{n}|q|^{n} \leq \sum_{n=0}^{\infty} c_{n} q_{0}^{n}$ with $q_{0}=e^{2 \pi i \tau_{0}}=e^{-\pi}$ and $\tau_{0}=i / 2$. The right-hand side of the inequality is equal to $j\left(\tau_{0}\right)-q_{0}^{-1}=66^{3}-e^{\pi} \leq 287473$. Note that we have used $j\left(\tau_{0}\right)=j\left(-1 / \tau_{0}\right)$ since the $j$-function is $\mathrm{SL}_{2}(\mathbb{Z})$-invariant, and that $j\left(-1 / \tau_{0}\right)=j(2 i)=$ $66^{3}$ by Table 12.20 in Cox11. 
In Lemma 3.1 we proved that the $j$-function is real on the vertical and unit circle geodesics of the fundamental domain and on the imaginary axis. We can even say that the $j$-function is not real outside of this set, as the following statement shows.

Corollary 3.4 If $\tau \in \mathcal{F}$ with $\operatorname{Re}(\tau) \neq 0, \pm \frac{1}{2}$ and $|\tau|>1$, then $\operatorname{Im}(j(\tau)) \neq 0$. Moreover, $\operatorname{Im}(j(\tau))<0$ for $0<\operatorname{Re}(\tau)<1 / 2$ and $\operatorname{Im}(j(\tau))>0$ for $-1 / 2<\operatorname{Re}(\tau)<0$.

Proof. The proof is just an application of the intermediate value theorem. We use that $j$ is injective on $\mathcal{F}$. Assume $j(\tau)=R$ is real with $|\tau|>1$ and $-1 / 2<\operatorname{Re}(\tau)<0$ or $0<\operatorname{Re}(\tau)<1 / 2$. If $0 \leq R \leq 1728$, then $j\left(e^{i \theta}\right)=R$ for some $\pi / 3 \leq \theta \leq \pi / 2$ by the intermediate value theorem applied to the real function $t \mapsto j\left(e^{i t}\right)$. This is a contradiction to the injectivity of $j$ on $\mathcal{F}$ since $|\tau|>1$.

Assume $R \geq 1728$. By Lemma $3.1 j(i R) \geq 1728$ and applying Proposition 3.2 we have $j(i R) \geq e^{2 \pi R}-2079$. Thus $j(i R) \geq R$ and applying the intermediate value theorem again gives a $t \geq 1$ with $j(i t)=R$. This is a contradiction since $0<\tau<1 / 2$. The case when $R<0$ follows similarly.

To show $\operatorname{Im}(j(\tau))<0$ for $0<\operatorname{Re}(\tau)<1 / 2$ and $|\tau|>1$ we assume we have $\tau_{0}, \tau_{1}$ in the interior of the fundamental domain $\mathcal{F}^{\circ}$ with positive real part and such that $\operatorname{Im}\left(j\left(\tau_{0}\right)\right)<0$ and $\operatorname{Im}\left(j\left(\tau_{1}\right)\right)>0$. Choose a path in $\mathcal{F}^{\circ}$, parametrized by $\gamma:[0,1] \rightarrow \mathcal{F}$, such that $\gamma(0)=\tau_{0}, \gamma(1)=\tau_{1}$ and such that every point in $\gamma([0,1])$ is in the interior of $\mathcal{F}$ and has positive real part. The function $t \mapsto \operatorname{Im}(j(\gamma(t)))$ is continuous and satisfies $\operatorname{Im}(j(\gamma(0)))=\operatorname{Im}\left(j\left(\tau_{0}\right)\right)<0$ and $\operatorname{Im}(j(\gamma(1)))=\operatorname{Im}\left(j\left(\tau_{1}\right)\right)>0$. By the intermediate value theorem we have a $0<t<1$ with $\operatorname{Im}(j(\gamma(t)))=0$ which is impossible by the choice of $\gamma$ and the first claim of the corollary. So it suffices to give a value of $j(\tau)$ with $0<\operatorname{Re}(\tau)<1 / 2$ and $\operatorname{Im}(j(\tau))<0$. We have

$$
j\left(\frac{1+5 i}{4}\right)=-1728(\sqrt{5}-2)^{20}(3-2 \sqrt{\sqrt{5}} i)^{6}\left(238 \sqrt{5}+\frac{861}{2}+60 \sqrt{\sqrt{5}} i\right)^{3}
$$

by page 17 of Adl14. A computation with Sage shows $\operatorname{Im}\left(j\left(\frac{1+5 i}{4}\right)\right)<0$. We must have $\operatorname{Im}(j(\tau))>0$ for $-1 / 2<\operatorname{Re}(\tau)<0$ by the same argument and the fact that $j: \mathcal{F} \rightarrow \mathbb{C}$ is surjective. This completes the proof.

The following two lemmas for $h(j)$ can be found in [BHK18]. The proofs follow directly from the statements in that very paper with the inequality $h(j-\alpha) \geq h(j)-h(\alpha)-\log 2$. For details see Proposition 4.1 and Proposition 4.3 in [BHK18].

Lemma 3.5 We have $[\mathbb{Q}(j): \mathbb{Q}]=\mathcal{C}(\Delta)$, and if $|\Delta| \geq 16$, then

$$
h(j-\alpha) \geq \frac{\pi|\Delta|^{1 / 2}-0.01}{\mathcal{C}(\Delta)}-h(\alpha)-\log 2 .
$$

Proof. The first statement is a classical result, see for example Chapter 13 in [Cox11]. The rest follows from Proposition 4.1 in BHK18] and $h(j)=h(j-\alpha+\alpha) \leq h(j-\alpha)+$ $h(\alpha)+\log 2$. 
Lemma 3.6 We have

$$
h(j-\alpha) \geq \frac{3}{\sqrt{5}} \log |\Delta|-9.79-h(\alpha)-\log 2 .
$$

This lemma is more delicate and follows from work of Colmez [Col98] and NakkajimaTaguchi [NT91]. The two previous lemmas bound the height of $j-\alpha$ from below. Next we want to bound the height of $j-\alpha$ from above when $j-\alpha$ is an algebraic unit.

The following lemma says that if two points in the fundamental domain are close together, then the difference of the images under the $j$-function can be bound from below in terms of the difference of the points. Recall that $\zeta=e^{2 \pi i / 6}$.

Lemma 3.7 Let $\zeta, \zeta^{2} \neq \xi \in \overline{\mathcal{F}}$. Put $B=4 \cdot 10^{5} \max \{1,|j(\xi)|\}$ and $A=\left|j^{\prime \prime}(i)\right|$ in the case when $\xi=i$ and $A=\left|j^{\prime}(\xi)\right|$ otherwise. For $|\tau-\xi| \leq \frac{A}{12 A+108 B} \leq \frac{1}{3}$ we have

$$
|j(\tau)-j(\xi)| \geq \frac{A}{4}|\tau-\xi|^{2} .
$$

If $\xi \neq i$ we even have

$$
|j(\tau)-j(\xi)| \geq \frac{A}{2}|\tau-\xi|
$$

for $|\tau-\xi| \leq \frac{A}{6 A+18 B}$.

Note that we can write $j^{\prime \prime}(i)=-2 \cdot 3^{4} \Gamma(1 / 4)^{8} / \pi^{4}$ as Kühne shows in the appendix of Wüs14. Since $\Gamma(1 / 4)=3.6256 \ldots>3$ we could further estimate the first of the two bounds in the lemma by

$$
|j(\tau)-1728| \geq 12413|\tau-i|^{2} .
$$

Proof. This is a special case of Lemma 2.4 in BLP16]. We take $f(\tau)=j(\tau)-j(\xi)$. Assume $|\tau-\xi| \leq 1 / 3$. Then by Lemma 3.3

$$
\begin{aligned}
|f(\tau)| & \leq|j(\tau)|+|j(\xi)| \leq|j(\xi)|+e^{2 \pi \operatorname{Im}(\tau)}+287473 \\
& \leq|j(\xi)|+e^{2 \pi(\operatorname{Im}(\xi)+1 / 3)}+287473 .
\end{aligned}
$$

We have $e^{2 \pi / 3}<9$ so applying Proposition 3.2 we obtain

$$
\begin{aligned}
|f(\tau)| & \leq|j(\xi)|+e^{2 \pi(\operatorname{Im}(\xi)+1 / 3)}+287473 \\
& \leq|j(\xi)|+9|j(\xi)|+9 \cdot 2079+287473 \\
& \leq 10|j(\xi)|+306184 \\
& \leq 4 \cdot 10^{5} \max \{1,|j(\xi)|\} .
\end{aligned}
$$

We treat the two cases $\xi=i$ and $\xi \neq i$ separately and start with the latter. By BLP16 we have

$$
|j(\tau)-j(\xi)|-A|\tau-\xi| \geq-\frac{A / 3+B}{(1 / 3)^{2}}|\tau-\xi|^{2}=-(3 A+9 B)|\tau-\xi|^{2},
$$


where $A=\left|j^{\prime}(\xi)\right|$. In the smaller disc $|\tau-\xi| \leq \frac{A}{2 \cdot(3 A+9 B)}$ we then obtain

$$
|j(\tau)-j(\xi)| \geq \frac{A}{2}|\tau-\xi| .
$$

Now assume $\xi=i$ and put $A=\left|j^{\prime \prime}(i)\right|$. By [BLP16] we conclude

$$
|j(\tau)-j(\xi)|-\frac{A}{2}|\tau-\xi|^{2} \geq-\frac{A / 9+B}{(1 / 3)^{3}}|\tau-\xi|^{3}=-(3 A+27 B)|\tau-\xi|^{3}
$$

and thus

$$
|j(\tau)-j(\xi)| \geq \frac{A}{4}|\tau-\xi|^{2} .
$$

in the smaller disc $|\tau-\xi| \leq \frac{A}{4 \cdot(3 A+27 B)}$.

We can also bound the $j$-invariants outside of a neighborhood of $\xi$ as the following lemma shows. We write

$$
\mathcal{F}_{+}=\{\tau \in \mathcal{F} ; 0 \leq \operatorname{Re}(\tau) \leq 1 / 2\}
$$

and

$$
\mathcal{F}_{-}=\{\tau \in \mathcal{F} ;-1 / 2 \leq \operatorname{Re}(\tau) \leq 0\} .
$$

Lemma 3.8 Let $\zeta \neq \xi, \tau \in \mathcal{F}_{+}$and put $A=\left|j^{\prime \prime}(i)\right|$ if $\xi=i$ and $A=\left|j^{\prime}(\xi)\right|$ otherwise. Also define $B=4 \cdot 10^{5} \max \{1,|j(\xi)|\}$. Assume $|\tau-\xi| \geq \delta$, where $\delta$ is defined as the minimum of $\frac{A}{12 A+108 B}$ and half the (euclidean) distance of $\xi$ to any geodesic of $\partial \mathcal{F}_{+}$ (i.e. the vertical line segments with real part 0 and $1 / 2$ and the part of the unit circle between those lines) not containing $\xi$. Then

$$
|j(\tau)-j(\xi)| \geq c(\xi)
$$

where $c(\xi)>0$ is a constant depending on $\xi$ and is given by

$$
c(\xi)= \begin{cases}A \delta / 2 & \text { if } \xi \in \partial \mathcal{F}_{+} \backslash\{i\} \\ A \delta^{2} / 4 & \text { if } \xi=i \\ \min \{|\operatorname{Im}(j(\xi))|, A \delta / 2\} & \text { otherwise. }\end{cases}
$$

Proof. We want to apply the maximum modulus principle. To do this we will give lower bounds on the boundary of $\mathcal{F}_{+}$, see Figure 1 . By the previous lemma we have

$$
|j(\tau)-j(\xi)| \geq \frac{A}{2} \delta
$$

or

$$
|j(\tau)-j(i)| \geq \frac{A}{4} \delta^{2}
$$




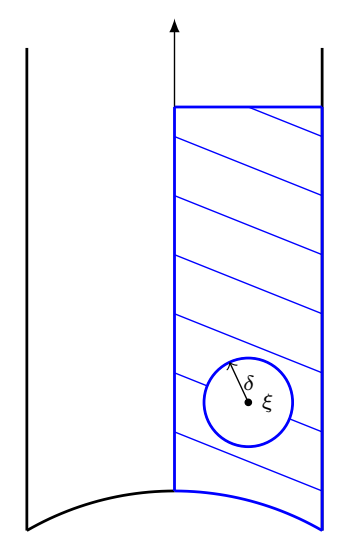

Figure 1: Application of the maximum modulus principle to the blue area.

on the circle $|\tau-\xi|=\delta$. Also $\operatorname{Im}(\tau) \geq 6 \operatorname{Im}(\xi)$ implies $\operatorname{Im}(\tau) \geq \operatorname{Im}(\xi)+1$, so we obtain by applying Proposition 3.2 twice

$$
\begin{aligned}
|j(\xi)| & \leq 2079+e^{2 \pi \operatorname{Im}(\xi)}=2079-20 e^{2 \pi \frac{\sqrt{3}}{2}}+20 e^{2 \pi \frac{\sqrt{3}}{2}}+e^{2 \pi \operatorname{Im}(\xi)} \\
& <-2079+20 e^{2 \pi \frac{\sqrt{3}}{2}}+e^{2 \pi \operatorname{Im}(\xi)} \leq-2079+20 e^{2 \pi \operatorname{Im}(\xi)}+e^{2 \pi \operatorname{Im}(\xi)} \\
& \leq-2079+21 e^{2 \pi \operatorname{Im}(\xi)}<-2079+e^{2 \pi} e^{2 \pi \operatorname{Im}(\xi)} \\
& \leq-2079+e^{2 \pi \operatorname{Im}(\tau)} \\
& \leq|j(\tau)| .
\end{aligned}
$$

Thus, by using Proposition 3.2 twice we get

$$
\begin{aligned}
|j(\tau)-j(\xi)| & \geq|j(\tau)|-|j(\xi)| \\
& \geq-2079+e^{2 \pi \operatorname{Im}(\tau)}-\left(2079+e^{2 \pi \operatorname{Im}(\xi)}\right) \\
& \geq-2 \cdot 2079+e^{2 \pi \operatorname{Im}(\tau)}-e^{2 \pi \operatorname{Im}(\xi)} .
\end{aligned}
$$

Now we use $\operatorname{Im}(\tau) \geq 6 \operatorname{Im}(\xi)$ to get

$$
|j(\tau)-j(\xi)| \geq-2 \cdot 2079+e^{12 \pi \operatorname{Im}(\xi)}-e^{2 \pi \operatorname{Im}(\xi)} .
$$

We have $e^{2 x} \geq 2 e^{x}$ for any $x \geq \log 2$ and $2 \pi \operatorname{Im}(\xi) \geq 1$ so that

$$
\begin{aligned}
|j(\tau)-j(\xi)| & \geq-2 \cdot 2079+e^{4 \pi \operatorname{Im}(\xi)}+e^{2 \pi \operatorname{Im}(\xi)}+e^{4 \pi \operatorname{Im}(\xi)}-e^{2 \pi \operatorname{Im}(\xi)} \\
& =-2 \cdot 2079+e^{4 \pi \operatorname{Im}(\xi)}+e^{4 \pi \operatorname{Im}(\xi)} .
\end{aligned}
$$

But also because of $\operatorname{Im}(\xi) \geq \sqrt{3} / 2$ we get

$$
|j(\tau)-j(\xi)| \geq e^{4 \pi \operatorname{Im}(\xi)} .
$$


We have $\delta \leq 1 / 12$ by definition and Lemme 1 of [FP87] gives for $\xi \neq i$

$$
\begin{aligned}
\frac{A}{2} \delta & \leq \frac{A}{24} \leq \frac{8 \pi}{24} e^{2(\pi+1) \max \left\{\operatorname{Im}(\xi), \operatorname{Im}(\xi)^{-1}\right\}} \\
& \leq \frac{8 \pi}{24} e^{3 \pi \max \left\{\operatorname{Im}(\xi), \operatorname{Im}(\xi)^{-1}\right\}} .
\end{aligned}
$$

So if $\operatorname{Im}(\xi) \geq 1$, then

$$
|j(\tau)-j(\xi)| \geq \frac{A}{2} \delta .
$$

If $\sqrt{3} / 2 \leq \operatorname{Im}(\xi) \leq 1$, then

$$
\frac{A}{2} \delta \leq \frac{8 \pi}{24} e^{3 \pi \operatorname{Im}(\xi)^{-1}} \leq \frac{8 \pi}{24} e^{2 \pi / \sqrt{3}} \leq e^{4 \pi \cdot \sqrt{3} / 2} \leq e^{4 \pi \operatorname{Im}(\xi)} .
$$

So in any case

$$
|j(\tau)-j(\xi)| \geq \frac{A}{2} \delta
$$

for $\xi \neq i$. If $\xi=i$, then as described after Lemma 3.7 we get

$$
\frac{A}{4} \delta^{2} \leq 12414 \delta^{2} \leq 87
$$

which together with (6) gives

$$
|j(\tau)-j(i)| \geq \frac{A}{4} \delta^{2}
$$

So we have treated all the $\tau$ with large imaginary part. Now we have to go through the different cases for $\xi$ to bound the boundary. We start with $\operatorname{Re}(\xi)=1 / 2$ so that we are in the following case.

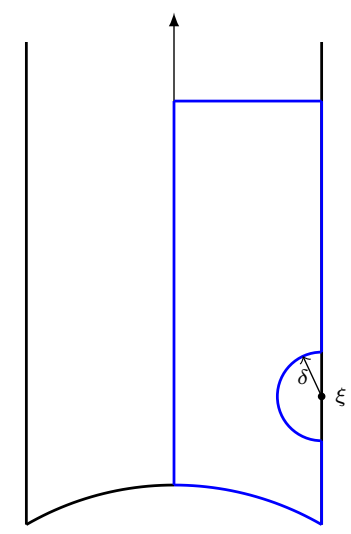

For the remainder of the proof we will be using that $j$ is monotonically increasing or decreasing on the boundary as shown in Lemma 3.1. If $\tau$ is on the same boundary 
component as $\xi$, then $\operatorname{Im}(\tau) \geq \operatorname{Im}(\xi)+\delta$ or $\operatorname{Im}(\tau) \leq \operatorname{Im}(\xi)-\delta$. Therefore, by monotonicity either $|j(\tau)|>|j(\xi)|$ which implies

$$
|j(\tau)-j(\xi)| \geq j(\xi)-j(\tau) \geq j(\xi)-j(\xi+i \delta)=|j(\xi)-j(\xi+i \delta)| \geq \frac{A}{2} \delta
$$

or $|j(\tau)|<|j(\xi)|$ and

$$
|j(\tau)-j(\xi)| \geq|j(\xi)|-|j(\tau)| \geq j(\xi-i \delta)-j(\xi)=|j(\xi)-j(\xi-i \delta)| \geq \frac{A}{2} \delta .
$$

Note that the last inequality of both displays follows from Lemma 3.7 on the boundary $|\tau-\xi|=\delta$. If $|\tau|=1$ or $\operatorname{Re}(\tau)=0$, then $j(\tau) \geq 0$ and $j(\xi)<0$, so that

$$
\begin{aligned}
|j(\tau)-j(\xi)| & =j(\tau)-j(\xi) \geq-j(\xi) \\
& \geq j(\xi-i \delta)-j(\xi)=|j(\xi)-j(\xi-i \delta)| \\
& \geq \frac{A}{2} \delta .
\end{aligned}
$$

Here we have used Lemma 3.7 for the last estimate. Altogether, if $\operatorname{Re}(\xi)=1 / 2$ we get by the minimum modulus principle

$$
|j(\tau)-j(\xi)| \geq \frac{A}{2} \delta
$$

as desired.

The second case is when $\operatorname{Re}(\xi)=0$ and $\xi \neq i$. We have $j(\tau) \leq 0$ if $\operatorname{Re}(\tau)=1 / 2$, and thus

$$
\begin{aligned}
|j(\xi)-j(\tau)| & \geq j(\xi) \geq j(\xi)-j(\xi-i \delta) \\
& =|j(\xi)-j(\xi-i \delta)| \\
& \geq \frac{A}{2} \delta .
\end{aligned}
$$

If $|\tau|=1$, then again by monotonicity and Lemma 3.7

$$
\begin{aligned}
|j(\xi)-j(\tau)| & \geq j(\xi)-j(\tau) \geq j(\xi)-j(\xi-i \delta) \\
& =|j(\xi)-j(\xi-i \delta)| \\
& \geq \frac{A}{2} \delta .
\end{aligned}
$$

For the case when $\operatorname{Re}(\tau)=0$ we have two subcases. When $\operatorname{Im}(\tau) \geq \operatorname{Im}(\xi)+\delta$, it follows $j(\tau)>j(\xi)$ and hence

$$
|j(\xi)-j(\tau)| \geq j(\xi+i \delta)-j(\xi) \geq \frac{A}{2} \delta .
$$

Or we have $\operatorname{Im}(\tau) \leq \operatorname{Im}(\xi)-\delta$ and we get

$$
|j(\xi)-j(\tau)| \geq j(\xi)-j(\xi-i \delta) \geq \frac{A}{2} \delta .
$$


In sum, by applying the minimum modulus principle we obtain

$$
|j(\tau)-j(\xi)| \geq \frac{A}{2} \delta .
$$

The third case is $|\xi|=1$ and $\xi \neq i$. Write $\xi=e^{i \theta}$. Again we have three subcases. If $\operatorname{Re}(\tau)=1 / 2$, then $j(\tau) \leq 0$ and

$$
\begin{aligned}
|j(\xi)-j(\tau)| & \geq j(\xi)-j(\tau) \geq j(\xi) \\
& \geq j(\xi)-j\left(e^{i(\theta-2 \arcsin (\delta / 2))}\right) \\
& =\left|j(\xi)-j\left(e^{i(\theta-2 \arcsin (\delta / 2))}\right)\right| \\
& \geq \frac{A}{2} \delta .
\end{aligned}
$$

Note that $e^{i(\theta-2 \arcsin (\delta / 2))}$ is one of the two points where the circle of radius $\delta$ and the unit circle intersect. If $\operatorname{Re}(\tau)=0$, then $j(\tau) \geq 1728>j\left(e^{i(\theta+2 \arcsin (\delta / 2))}\right)>j(\xi)$ and

$$
\begin{aligned}
|j(\xi)-j(\tau)| & \geq j(\tau)-j(\xi) \geq j\left(e^{i(\theta+2 \arcsin (\delta / 2))}\right)-j(\xi) \\
& =\left|j(\xi)-j\left(e^{i(\theta+2 \arcsin (\delta / 2))}\right)\right| \\
& \geq \frac{A}{2} \delta .
\end{aligned}
$$

If $|\tau|=1$, then $j(\tau)<j(\xi)$ or $j(\tau)>j(\xi)$ and Lemma 3.7 tells us

$$
|j(\xi)-j(\tau)| \geq\left|j(\xi)-j\left(e^{i(\theta \pm 2 \arcsin (\delta / 2))}\right)\right| \geq \frac{A}{2} \delta .
$$

By applying the minimum modulus principle we obtain the same result as in equation (8).

If $\xi=i$, then the estimates in equations (7) and (10) hold with $A \delta / 2$ replaced by $A \delta^{2} / 4$. Also equation (9) holds with $\xi$ replaced by $i$ and $A \delta / 2$ replaced by $A \delta^{2} / 4$, and thus by the minimum modulus principle

$$
|j(\tau)-j(i)| \geq \frac{A}{4} \delta^{2} .
$$

The last case is $0<\operatorname{Re}(\xi)<1 / 2$ and $|\xi|>1$. Let $\tau \in \partial \mathcal{F}_{+}$. Then $j(\tau)$ is real and we have

$$
|j(\xi)-j(\tau)| \geq|\operatorname{Im}(j(\xi))-\operatorname{Im}(j(\tau))|=|\operatorname{Im}(j(\xi))| .
$$

This is the case shown in Figure 1. Applying the minimum modulus principle gives the desired result.

Note that the same claim holds for $\mathcal{F}_{-}$since we have the symmetry $\operatorname{Re}(j(x+i y))=$ $\operatorname{Re}(j(-x+i y))$ and $\operatorname{Im}(j(x+i y))=-\operatorname{Im}(j(-x+i y))$ which directly follows from the $q$-expansion. If $j(\tau)$ and $j(\xi)$ are close we want $\left|\tau_{\sigma}-\xi_{\sigma}\right|$ to be small too, but we can not get this in general as Figure 2 shows. 


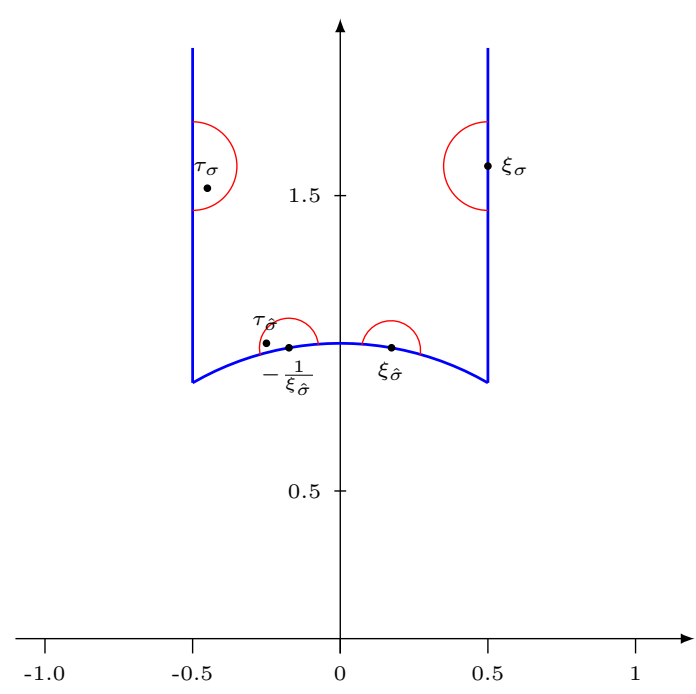

Figure 2: Neighborhoods of points on the boundary

Lemma 3.9 In the same setting as in the previous lemma, if $|j(\tau)-j(\xi)|<c(\xi)$, then $|\tau-M \xi|<\delta$ with $M \xi \in \overline{\mathcal{F}}$ for some $M \in \mathcal{T}$ where $\mathcal{T}=\left\{\left(\begin{array}{cc}1 & 0 \\ 0 & 1\end{array}\right),\left(\begin{array}{cc}1 & \pm 1 \\ 0 & 1\end{array}\right),\left(\begin{array}{cc}0 & -1 \\ 1 & 0\end{array}\right)\right\}$.

Proof. If $\xi, \tau$ are both in $\mathcal{F}_{+}$or both in $\mathcal{F}_{-}$, then $|\tau-\xi| \leq \delta$ by Lemma 3.8. We now can assume without loss of generality $\operatorname{Re}(\xi)<0$ and $\operatorname{Re}(\tau)>0$. If $\xi$ is on the boundary of $\mathcal{F}$, then $\operatorname{Re}(\xi)=-1 / 2$. Then we can apply Lemma 3.8 to $\tau$ and $\left(\begin{array}{ll}1 & 1 \\ 0 & 1\end{array}\right) \xi$ and use $j(\xi)=j(\xi+1)$. If $|\xi|=1$ and $\operatorname{Re}(\xi)<0$, then we can again apply Lemma 3.8 to $\tau$ and $\left(\begin{array}{cc}0 & -1 \\ 1 & 0\end{array}\right) \xi$ and use $j(\xi)=j\left(\left(\begin{array}{cc}0 & -1 \\ 1 & 0\end{array}\right) \xi\right)$. If $-1 / 2<\operatorname{Re}(\xi)<0$ and $\tau \in \mathcal{F}_{+}$, then by Corollary 3.4

$$
\begin{aligned}
|j(\xi)-j(\tau)| & \geq|\operatorname{Im}(j(\xi))-\operatorname{Im}(j(\tau))|=\operatorname{Im}(j(\xi))-\operatorname{Im}(j(\tau)) \\
& =\operatorname{Im}(j(\xi))+|\operatorname{Im}(j(\tau))| \\
& \geq \operatorname{Im}(j(\xi)) \\
& \geq c(\xi)
\end{aligned}
$$

contrary to the assumption.

The following lemma can be found in Hab15 as Lemma 5.

Lemma 3.10 Let $\sigma: \overline{\mathbb{Q}} \hookrightarrow \mathbb{C}$ and let $\tau$ be imaginary quadratic. Let $\tau_{\sigma}$ satisfy $j\left(\tau_{\sigma}\right)=$ $\sigma(j(\tau))$ with $\tau_{\sigma} \in \mathcal{F}$. If $\Delta$ is the discriminant of the endomorphism ring associated to $j(\tau)$, then $\tau_{\sigma}$ is imaginary quadratic and $h\left(\tau_{\sigma}\right) \leq \log \sqrt{|\Delta|}$.

With these lemmas we are now able to bound the height from above using linear forms in logarithms. Instead of using a result by Masser Mas75] we are going to use a result by David [Dav95] to make the constants explicit. We are going to use this lemma to prove 
a result on linear forms. It is a special case of Théorème 2.1 in Dav95]. The height of a matrix in $\mathrm{SL}_{2}(\mathbb{Z})$ will be the height when regarded as a member of $\mathbb{Q}^{4}$.

Lemma 3.11 Let $\tau \in \mathbb{H}$ be imaginary quadratic and let $E: y^{2}=4 x^{3}-g_{2} x-g_{3}$ be an elliptic curve without complex multiplication such that $E(\mathbb{C}) \simeq \mathbb{C} /\left(\omega_{1} \mathbb{Z}+\omega_{2} \mathbb{Z}\right)$ with $\xi=\omega_{2} / \omega_{1} \in \mathcal{F}$. Assume $E$ is defined over a number field of degree D. Put $h=\max \left\{1, h\left(1, g_{2}, g_{3}\right), h(j(\xi))\right\}$ and assume $\Delta$ is the discriminant of the endomorphism ring of an elliptic curve with $j$-invariant $j(\tau)$. Pick any $M=\left(\begin{array}{ll}\alpha & \beta \\ \gamma & \delta\end{array}\right) \in \mathrm{SL}_{2}(\mathbb{Z})$. If $|\Delta| \geq \max \left\{2 D, e^{12 \pi h}, 4 H(M)^{4}\right\}$, then we have

$$
\log \left|(\gamma \tau-\alpha) \omega_{2}-(\delta \tau-\beta) \omega_{1}\right| \geq-c_{2}(\log |\Delta|)^{4}
$$

with

$$
c_{2}=2^{50} 3^{43} 5^{18} \cdot D^{6} \cdot h^{2} .
$$

Proof. We follow the notation in Dav95. Put $\mathcal{L}\left(z_{0}, z_{1}, z_{2}\right)=(\gamma \tau-\alpha) z_{1}-(\delta \tau-\beta) z_{2}$. We choose the variables $u_{1}, u_{2}$ in the theorem to be $\omega_{2}$ and $\omega_{1}$, respectively. Then $\gamma_{1}=\gamma_{2}=(0,0,1)$ and $v=\left(1, \omega_{2}, \omega_{1}\right)$. Note that $\mathcal{L}(v) \neq 0$ since otherwise we would have $\tau=M .\left(\omega_{2} / \omega_{1}\right)=M \xi$ but $\xi$ and thus $M \xi$ are not imaginary quadratic. We have to estimate the height of the coefficients of the linear form. By Lemma 3.10 we have $h(\tau) \leq \log \sqrt{|\Delta|}$. Thus the multiplicative height of the coefficients can be estimated by

$$
\begin{aligned}
H(\gamma \tau-\beta) & \leq 2 H(\gamma \tau) H(\beta) \leq 2 H(\gamma) H(\tau) H(\beta) \leq 2 H(M)^{2} H(\tau) \\
& \leq 2 H(M)^{2}|\Delta|^{1 / 2} \leq|\Delta|
\end{aligned}
$$

and similarly

$$
H(\delta \tau-\beta) \leq|\Delta|
$$

We thus can work with $B=|\Delta|$ which satisfies condition (2) of Théorème 2.1. Moreover, we can pick $V=V_{1}=V_{2}=e^{12 \pi h}$. To see this note

$$
\frac{3 \pi\left|u_{1}\right|^{2}}{\left|\omega_{1}\right|^{2} \operatorname{Im}(\xi) 2 D} \leq \frac{3 \pi\left|u_{1}\right|^{2}}{\left|\omega_{1}\right|^{2} \operatorname{Im}(\xi) D} \leq \frac{3 \pi|\xi|^{2}}{\operatorname{Im}(\xi) D} \leq 4 \pi \frac{|\xi|}{D}
$$

and also

$$
\frac{3 \pi\left|u_{2}\right|^{2}}{\left|\omega_{1}\right|^{2} \operatorname{Im}(\xi) D} \leq \frac{3 \pi}{\operatorname{Im}(\xi) D} \leq 4 \pi .
$$

By Lemme 1 item (iv) in [FP87] we have

$$
|\xi| \leq \frac{3}{2} \log \max \{e,|j(\xi)|\}
$$


and therefore

$$
\begin{aligned}
\frac{|\xi|}{D} & \leq \frac{3}{2} \frac{1}{D} \log \max \{e,|j(\xi)|\} \\
& \leq \frac{3}{2} \frac{1}{D}(1+\log \max \{1,|j(\xi)|\}) \\
& \leq \frac{3}{2} \frac{1}{D}\left(1+\sum_{\nu \in M_{K}} d_{\nu} \log \max \left\{1,|j(\xi)|_{\nu}\right\}\right) \\
& \leq \frac{3}{2}+\frac{3}{2} h(j(\xi)) .
\end{aligned}
$$

Here $K$ denotes the field of definition for $E$. Thus conditions (1) and (3) of the theorem in Dav95 are satisfied. We thus can apply the theorem and get the lower bound

$\log |\mathcal{L}(v)| \geq-C \cdot 2^{6} D^{6}(\log B+\log (2 D)+1) \cdot(\log \log B+h+\log (2 D)+1)^{3} \log V_{1} \log V_{2}$ $\geq-2^{16} 3^{3} C \cdot D^{6} \cdot h^{2} \cdot(\log B)^{4}$

since $h \leq \log B$ and $\log (2 D) \leq \log B$. The constant $C$ comes from Dav95 and is given by

$$
C=10^{18} \cdot 4^{8} \cdot 3^{40} .
$$

This gives the desired inequality.

For the following, if $\tau \in \mathcal{F}$ with $j(\tau)$ algebraic and a field embedding $\sigma: \mathbb{Q}(\alpha) \hookrightarrow \mathbb{C}$, $\alpha \in \overline{\mathbb{Q}}$, are given, then $\tau_{\sigma} \in \mathcal{F}$ is defined by $\sigma(j(\tau))=j\left(\tau_{\sigma}\right)$. Note that for fixed $\xi \in \mathcal{F}$ different from $\zeta, \zeta^{2}, i$ we have $j^{\prime}\left(\xi_{\sigma}\right) \neq 0$. Suppose that $j(\xi)=\alpha$ is algebraic. We define the function

$$
\mathcal{P}(\xi)=\log \max _{\sigma}\left\{1, c\left(\xi_{\sigma}\right)^{-1}\right\},
$$

where $\sigma$ runs over all embeddings $\sigma: \mathbb{Q}(\alpha) \hookrightarrow \mathbb{C}$, and $c\left(\xi_{\sigma}\right)$ is defined as in Lemma 3.8 Note that the expression in the maximum is larger than 12 since $\delta_{\sigma} \leq 1 / 12$, and hence $\mathcal{P}(\xi)>0$. The function is large when some $\xi_{\sigma}$ is close to one of the three points $i, \zeta, \zeta^{2}$ or $\xi_{\sigma}$ is close to the boundary of $\mathcal{F}$ or to the vertical imaginary axis.

Proposition 3.12 Assume $j$ is a singular modulus. Let $\alpha=j(\xi), \xi \in \mathcal{F}$, be an algebraic number that is the $j$-invariant of an elliptic curve without complex multiplication. Let $E: y^{2}=4 x^{3}-g_{2} x-g_{3}$ be a model for $\alpha$ such that $E(\mathbb{C}) \simeq \mathbb{C} /\left(\omega_{1} \mathbb{Z}+\omega_{2} \mathbb{Z}\right)$ and $\xi=\omega_{2} / \omega_{1}$. Similarly, choose a model for $E^{\sigma}$ for any $\sigma: \mathbb{Q}(\alpha) \hookrightarrow \mathbb{C}$. Further assume that $E$ is defined over a field of degree $D$ and $|\Delta| \geq \max \left\{2 D, e^{12 \pi h}\right\}$. Assume that $j-\alpha$ is an algebraic unit and let $0<\varepsilon<1 / 4$. We can bound the height by

$$
h(j-\alpha) \leq c_{2} \frac{\sum_{\sigma: \mathbb{Q}(j, \alpha) \rightarrow \mathbb{C}} \sum_{M \in \mathcal{T}} \mathcal{C}\left(\Delta ; M \xi_{\sigma} ; \varepsilon\right)}{16 \cdot \mathcal{C}(\Delta)}(\log |\Delta|)^{4}+5 \mathcal{P}(\xi)+4 \mathcal{M}(\xi)+|\log \varepsilon|,
$$

where $\mathcal{T}=\left\{\left(\begin{array}{ll}1 & 0 \\ 0 & 1\end{array}\right),\left(\begin{array}{cc}1 & \pm 1 \\ 0 & 1\end{array}\right),\left(\begin{array}{cc}0 & -1 \\ 1 & 0\end{array}\right)\right\}$ and $\mathcal{M}(\xi)=\log \max _{\sigma}\left\{1,\left|\omega_{\sigma, 1}\right|,\left|\omega_{\sigma, 2}\right|\right\}$. 
Proof. Let $j(\xi)=\alpha$. Since $j-\alpha$ is an algebraic unit the height can be computed by

$$
h(j-\alpha)=\frac{1}{[\mathbb{Q}(j, \alpha): \mathbb{Q}]} \sum_{\sigma} \log \max \left\{1,\left|\sigma(j-\alpha)^{-1}\right|\right\},
$$

where $\sigma$ runs over all field embeddings $\sigma: \mathbb{Q}(j, \alpha) \rightarrow \mathbb{C}$. Let

$$
\varepsilon_{0}=\varepsilon \cdot \min _{\sigma: \mathbb{Q}(\alpha) \rightarrow \mathbb{C}}\left\{1, c\left(\xi_{\sigma}\right)\right\} .
$$

We split the sum (12) into terms for which $|\sigma(j-\alpha)|<\varepsilon_{0}$ and those for which $1 \geq$ $|\sigma(j-\alpha)| \geq \varepsilon_{0}$.

Assume $|\sigma(j-\alpha)|<\varepsilon_{0}$. Thus $|\sigma(j-\alpha)|<c\left(\xi_{\sigma}\right)$. We want to show $\left|\tau_{\sigma}-M \xi_{\sigma}\right|<\varepsilon$ for some $M \in \mathcal{T}$. We can apply Lemma 3.9 to get $\left|\tau_{\sigma}-M \xi_{\sigma}\right|<\delta_{\sigma}$ for some $M \in \mathcal{T}$. By definition of $\delta_{\sigma}$ we have $\delta_{\sigma} \leq \frac{\left|j^{\prime}\left(\xi_{\sigma}\right)\right|}{6\left|j^{\prime}\left(\xi_{\sigma}\right)\right|+72 \cdot 10^{5} \max \left\{1,\left|j\left(\xi_{\sigma}\right)\right|\right\}}$, so that we can apply Lemma 3.7 and $j\left(\xi_{\sigma}\right)=j\left(M \xi_{\sigma}\right)$ to get

$$
\frac{\left|j^{\prime}\left(\xi_{\sigma}\right)\right|}{2}\left|\tau_{\sigma}-M \xi_{\sigma}\right| \leq\left|j\left(\tau_{\sigma}\right)-j\left(\xi_{\sigma}\right)\right|<\varepsilon_{0} \leq \varepsilon c\left(\xi_{\sigma}\right) \leq \varepsilon \frac{\left|j^{\prime}\left(\xi_{\sigma}\right)\right|}{2} \delta_{\sigma} \leq \varepsilon \frac{\left|j^{\prime}\left(\xi_{\sigma}\right)\right|}{2},
$$

which implies $\left|\tau_{\sigma}-M \xi_{\sigma}\right|<\varepsilon$. But we also get

$$
\left|j\left(\tau_{\sigma}\right)-j\left(\xi_{\sigma}\right)\right| \geq \frac{\left|j^{\prime}\left(\xi_{\sigma}\right)\right|}{2}\left|\tau_{\sigma}-M \xi_{\sigma}\right| .
$$

Note that the right-hand side is not 0 since $j\left(\xi_{\sigma}\right)$ does not have complex multiplication but $j\left(\tau_{\sigma}\right)$ does. The same argument tells us $j^{\prime}\left(\xi_{\sigma}\right) \neq 0$. Write $M=\left(\begin{array}{ll}\alpha & \beta \\ \gamma & \delta\end{array}\right)$. Then we get

$$
\begin{aligned}
\left|\tau_{\sigma}-M \xi_{\sigma}\right| & =\left|\tau_{\sigma}-M \frac{\omega_{\sigma, 2}}{\omega_{\sigma, 1}}\right| \\
& =\frac{1}{\left|\gamma \omega_{\sigma, 2}+\delta \omega_{\sigma, 1}\right|}\left|(\gamma \tau-\alpha) \omega_{\sigma, 2}+(\delta \tau-\beta) \omega_{\sigma, 1}\right| \\
& \geq \frac{1}{\max \left\{\left|\omega_{\sigma, 2}\right|,\left|\omega_{\sigma, 1}\right|\right\}}\left|(\gamma \tau-\alpha) \omega_{\sigma, 2}+(\delta \tau-\beta) \omega_{\sigma, 1}\right|
\end{aligned}
$$

since $\gamma$ and $\delta$ are not both 1 . Using this together with 13 and Lemma 3.11 we obtain

$$
\begin{aligned}
\log |\sigma(j-\alpha)| & =\log \left|j\left(\tau_{\sigma}\right)-j\left(\xi_{\sigma}\right)\right| \\
& \geq \log \frac{\left|j^{\prime}\left(\xi_{\sigma}\right)\right|}{2}+\log \min \left\{\left|\omega_{\sigma, 1}\right|^{-1},\left|\omega_{\sigma, 2}\right|^{-1}\right\}-c_{2}(\log |\Delta|)^{4} \\
& \geq \log \frac{\left|j^{\prime}\left(\xi_{\sigma}\right)\right|}{2}+\log \min \left\{1,\left|\omega_{\sigma, 1}\right|^{-1},\left|\omega_{\sigma, 2}\right|^{-1}\right\}-c_{2}(\log |\Delta|)^{4} .
\end{aligned}
$$

Since with $|\sigma(j-\alpha)|<\varepsilon_{0}$ we also have $\left|\tau_{\sigma}-M \xi_{\sigma}\right|<\varepsilon$ for some matrix $M \in \mathcal{T}$ as mentioned before, we obtain that $\tau_{\sigma}$ corresponds to a form in $\mathcal{C}\left(\Delta ; M \xi_{\sigma} ; \varepsilon\right)$. We have

$$
c\left(\xi_{\sigma}\right) \leq \frac{\left|j^{\prime}\left(\xi_{\sigma}\right)\right|}{2} \delta \leq \frac{\left|j^{\prime}\left(\xi_{\sigma}\right)\right|}{2}
$$


so that

$$
\log \max \left\{1, \frac{2}{\left|j^{\prime}\left(\xi_{\sigma}\right)\right|}\right\} \leq \mathcal{P}(\xi) .
$$

Thus, if we use (14) we get

$$
\begin{aligned}
h(j-\alpha) \leq & -\frac{1}{[\mathbb{Q}(j, \alpha): \mathbb{Q}]} \sum_{|\sigma(j-\alpha)|<\varepsilon_{0}} \log |\sigma(j-\alpha)|+\left|\log \varepsilon_{0}\right| \\
\leq & c_{2} \frac{\sum_{\sigma: \mathbb{Q}(\alpha) \hookrightarrow \mathbb{C}} \sum_{M \in \mathcal{T}} \mathcal{C}\left(\Delta ; M \xi_{\sigma} ; \varepsilon\right)}{16 \cdot[\mathbb{Q}(j, \alpha): \mathbb{Q}]}(\log |\Delta|)^{4} \\
& +4 \mathcal{P}(\xi)-4 \log \min _{\sigma}\left\{1,\left|\omega_{\sigma, 1}\right|^{-1},\left|\omega_{\sigma, 2}\right|^{-1}\right\}+\left|\log \varepsilon_{0}\right| .
\end{aligned}
$$

If we plug in the definition for $\varepsilon_{0}$ we obtain

$$
\begin{aligned}
h(j-\alpha) \leq & c_{2} \frac{\sum_{\sigma: \mathbb{Q}(\alpha) \hookrightarrow \mathbb{C}} \sum_{M \in \mathcal{T}} \mathcal{C}\left(\Delta ; M \xi_{\sigma} ; \varepsilon\right)}{16 \cdot \mathcal{C}(\Delta)}(\log |\Delta|)^{4}+4 \mathcal{P}(\xi) \\
& +4 \log \max _{\sigma}\left\{1,\left|\omega_{\sigma, 1}\right|,\left|\omega_{\sigma, 2}\right|\right\}-\log \min _{\sigma}\left\{1, c\left(\xi_{\sigma}\right)\right\}+|\log \varepsilon| \\
= & c_{2} \frac{\sum_{\sigma: \mathbb{Q}(\alpha) \hookrightarrow \mathbb{C}} \sum_{M \in \mathcal{T}} \mathcal{C}\left(\Delta ; M \xi_{\sigma} ; \varepsilon\right)}{16 \cdot \mathcal{C}(\Delta)}(\log |\Delta|)^{4}+5 \mathcal{P}(\xi) \\
& +4 \log \max _{\sigma}\left\{1,\left|\omega_{\sigma, 1}\right|,\left|\omega_{\sigma, 2}\right|\right\}+|\log \varepsilon|,
\end{aligned}
$$

where we also used the first claim of Lemma 3.5 for the inequality.

The following lemmas can be found in Section 3 of [BHK18] as Lemmas 3.5 and 3.6.

Lemma 3.13 Assume that $|\Delta| \geq 10^{14}$. Then we have $F(\Delta) \geq|\Delta|^{0.34 / \log \log \left(|\Delta|^{1 / 2}\right)}$ and $F(\Delta) \geq 18 \log \log \left(|\Delta|^{1 / 2}\right)$.

Lemma 3.14 For $\Delta \neq-3,-4$ we have $\mathcal{C}(\Delta) \leq \pi^{-1}|\Delta|^{1 / 2}(2+\log |\Delta|)$.

Proof. Theorem 10.1 in Hua12 says $\mathcal{C}(\Delta) \leq \frac{\omega|\Delta|^{1 / 2}}{2 \pi} K(d)$ where $K(d)$ can be bounded by $2+\log |\Delta|$ according to Theorem 14.3 in Hua12 and $\omega$ is the number of roots of unity in the imaginary quadratic order of discriminant $\Delta$. But since $\Delta \neq-3,-4$ we have $\omega=2$ and the result follows.

We define $E:=E(\Delta)=F(\Delta)(\log |\Delta|)^{4}$.

Corollary 3.15 Assume $j-\alpha$ is a unit and $\alpha=j(\xi)$ with $\xi \in \mathcal{F}$ and such that $\alpha$ is algebraic but not a singular modulus. For $|\Delta| \geq 10^{14}$ we have

$$
h(j-\alpha) \leq c_{2}[\mathbb{Q}(\alpha): \mathbb{Q}] \frac{E(\Delta)}{2 \mathcal{C}(\Delta)}+\log \frac{E(\Delta)|\Delta|^{1 / 2}}{\mathcal{C}(\Delta)}+C^{\prime}
$$

where $C^{\prime}$ is a constant depending on $\alpha$ and is given by

$$
C^{\prime}=4[\mathbb{Q}(\alpha): \mathbb{Q}] c_{2}+5 \mathcal{P}(\xi)+4 \mathcal{M}(\xi) .
$$


Proof. We can use the previous results together with the bound for $\mathcal{C}\left(\Delta ; \xi_{\sigma} ; \varepsilon\right)$ to bound the height $h(j-\alpha)$. Put $\varepsilon=\frac{\mathcal{C}(\Delta)}{F(\Delta)(\log |\Delta|)^{4}|\Delta|^{1 / 2}}$. Since we assume that $|\Delta| \geq 10^{14}$ we have $F(\Delta) \geq 256$ and we obtain together with Lemma 3.14

$$
\varepsilon \leq \frac{\pi^{-1}|\Delta|^{1 / 2}(2+\log |\Delta|)}{256(\log |\Delta|)^{4}|\Delta|^{1 / 2}} \leq \frac{1}{256 \pi} \frac{2+\log 10^{14}}{\log 10^{14}}<2 \cdot 10^{-3} .
$$

Thus we can apply Proposition 3.12 together with Corollary 2.2 and obtain

$$
\begin{aligned}
h(j-\alpha) \leq & {[\mathbb{Q}(\alpha): \mathbb{Q}] c_{2} \frac{4 F(\Delta)\left(32|\Delta|^{1 / 2} \varepsilon^{2} \log \log \left(|\Delta|^{1 / 2}\right)+11|\Delta|^{1 / 2} \varepsilon+2\right)}{16 \cdot \mathcal{C}(\Delta)}(\log |\Delta|)^{4} } \\
& +5 \mathcal{P}(\xi)+4 \mathcal{M}(\xi)+|\log \varepsilon| \\
= & {[\mathbb{Q}(\alpha): \mathbb{Q}] c_{2} E \frac{128|\Delta|^{1 / 2} \log \log \left(|\Delta|^{1 / 2}\right)}{16 \cdot \mathcal{C}(\Delta)}\left(\frac{\mathcal{C}(\Delta)}{F(\Delta)(\log |\Delta|)^{4}|\Delta|^{1 / 2}}\right)^{2} } \\
& +[\mathbb{Q}(\alpha): \mathbb{Q}] c_{2} E \frac{44|\Delta|^{1 / 2}}{16 \cdot \mathcal{C}(\Delta)} \frac{\mathcal{C}(\Delta)}{F(\Delta)(\log |\Delta|)^{4}|\Delta|^{1 / 2}} \\
& +[\mathbb{Q}(\alpha): \mathbb{Q}] c_{2} \frac{E}{2 \mathcal{C}(\Delta)} \\
& +5 \mathcal{P}(\xi)+4 \mathcal{M}(\xi)+\log \left(\frac{F(\Delta)(\log |\Delta|)^{4}|\Delta|^{1 / 2}}{\mathcal{C}(\Delta)}\right) .
\end{aligned}
$$

We continue the estimate by simplifying the terms to get

$$
\begin{aligned}
h(j-\alpha) \leq & {[\mathbb{Q}(\alpha): \mathbb{Q}] c_{2} \frac{8 \log \log \left(|\Delta|^{1 / 2}\right)}{F(\Delta)} \frac{\mathcal{C}(\Delta)}{(\log |\Delta|)^{4}|\Delta|^{1 / 2}} } \\
& +3[\mathbb{Q}(\alpha): \mathbb{Q}] c_{2}+[\mathbb{Q}(\alpha): \mathbb{Q}] c_{2} \frac{E}{2 \mathcal{C}(\Delta)} \\
& +5 \mathcal{P}(\xi)+4 \mathcal{M}(\xi)+\log \left(\frac{E|\Delta|^{1 / 2}}{\mathcal{C}(\Delta)}\right) .
\end{aligned}
$$

Now we apply Lemma 3.13 to see

$$
\begin{aligned}
h(j-\alpha) \leq & {[\mathbb{Q}(\alpha): \mathbb{Q}] c_{2} \frac{1}{2} \frac{\mathcal{C}(\Delta)}{(\log |\Delta|)^{4}|\Delta|^{1 / 2}} } \\
& +3[\mathbb{Q}(\alpha): \mathbb{Q}] c_{2}+[\mathbb{Q}(\alpha): \mathbb{Q}] c_{2} \frac{E}{2 \mathcal{C}(\Delta)} \\
& +5 \mathcal{P}(\xi)+4 \mathcal{M}(\xi)+\log \left(\frac{E|\Delta|^{1 / 2}}{\mathcal{C}(\Delta)}\right) .
\end{aligned}
$$


We continue the estimate using Lemma 3.14

$$
\begin{aligned}
h(j-\alpha) \leq & {[\mathbb{Q}(\alpha): \mathbb{Q}] c_{2} \frac{1}{2 \pi} \frac{|\Delta|^{1 / 2}(2+\log |\Delta|)}{(\log |\Delta|)^{4}|\Delta|^{1 / 2}} } \\
& +3[\mathbb{Q}(\alpha): \mathbb{Q}] c_{2}+[\mathbb{Q}(\alpha): \mathbb{Q}] c_{2} \frac{E}{2 \mathcal{C}(\Delta)} \\
& +5 \mathcal{P}(\xi)+4 \mathcal{M}(\xi)+\log \left(\frac{E|\Delta|^{1 / 2}}{\mathcal{C}(\Delta)}\right) .
\end{aligned}
$$

Simplifying again results into

$$
\begin{aligned}
h(j-\alpha) \leq & {[\mathbb{Q}(\alpha): \mathbb{Q}] c_{2} \frac{1}{2 \pi} \frac{(2+\log |\Delta|)}{(\log |\Delta|)^{4}} } \\
& +3[\mathbb{Q}(\alpha): \mathbb{Q}] c_{2}+[\mathbb{Q}(\alpha): \mathbb{Q}] c_{2} \frac{E}{2 \mathcal{C}(\Delta)} \\
& +5 \mathcal{P}(\xi)+4 \mathcal{M}(\xi)+\log \left(\frac{E|\Delta|^{1 / 2}}{\mathcal{C}(\Delta)}\right)
\end{aligned}
$$

The function $x \mapsto \frac{2+\log x}{(\log x)^{4}}$ is decreasing for $x>1$. Thus we can substitute $x=10^{14}$ to continue the bound and get

$$
\begin{aligned}
h(j-\alpha) \leq & {[\mathbb{Q}(\alpha): \mathbb{Q}] c_{2} \frac{1}{2 \pi} \frac{35}{32^{4}} } \\
& +3[\mathbb{Q}(\alpha): \mathbb{Q}] c_{2}+[\mathbb{Q}(\alpha): \mathbb{Q}] c_{2} \frac{E}{2 \mathcal{C}(\Delta)} \\
& +5 \mathcal{P}(\xi)+4 \mathcal{M}(\xi)+\log \left(\frac{E|\Delta|^{1 / 2}}{\mathcal{C}(\Delta)}\right)
\end{aligned}
$$

This gives the desired inequality.

\section{Proof of the main theorem}

We now want to bound $\Delta$ to complete the main proof. We will do this by using the lower and upper bounds we derived in the last section. Throughout this section we assume $|\Delta| \geq 10^{50}$.

Put

$$
C=C^{\prime}+h(\alpha)+\log 2+0.01 .
$$

Combining the lower bounds for $h(j-\alpha)$ from Lemmas 3.5 and 3.6 with the upper bound from Corollary 3.15 we obtain the inequality

$$
L:=\max \left\{\pi \frac{|\Delta|^{1 / 2}}{\mathcal{C}(\Delta)}, \frac{3}{\sqrt{5}} \log |\Delta|-10\right\} \leq[\mathbb{Q}(\alpha): \mathbb{Q}] c_{2} \frac{E(\Delta)}{2 \mathcal{C}(\Delta)}+\log \frac{E(\Delta)|\Delta|^{1 / 2}}{\mathcal{C}(\Delta)}+C
$$


or equivalently

$$
1 \leq[\mathbb{Q}(\alpha): \mathbb{Q}] c_{2} \frac{E}{2 L \cdot \mathcal{C}(\Delta)}+\frac{\log E+C}{L}+\frac{\log \left(|\Delta|^{1 / 2} / \mathcal{C}(\Delta)\right)}{L} .
$$

For the remainder we assume that $|\Delta|$ is large enough so that $\log E+C \geq 0$. By Lemma 3.13 this is the case when $|\Delta| \geq e^{e^{e^{-C} / 18}}$. This in turn is true whenever $|\Delta| \geq 3$. Since $\frac{3}{\sqrt{5}} \log |\Delta|-10>0$ for $|\Delta| \geq 10^{14}$ this allows us to replace $L$ by $\frac{3}{\sqrt{5}} \log |\Delta|-10$ in the middle term. Similarly we can replace $L$ in the first term by $\pi|\Delta|^{1 / 2} / \mathcal{C}(\Delta)$ and obtain

$$
\begin{aligned}
1 & \leq[\mathbb{Q}(\alpha): \mathbb{Q}] c_{2} \frac{E}{2 \pi|\Delta|^{1 / 2}}+\frac{\log E+C}{\frac{3}{\sqrt{5}} \log |\Delta|-10}+\frac{\log \left(|\Delta|^{1 / 2} / \mathcal{C}(\Delta)\right)}{L} \\
& \leq[\mathbb{Q}(\alpha): \mathbb{Q}] c_{2} \frac{E}{2 \pi|\Delta|^{1 / 2}}+\frac{\log E}{\frac{3}{\sqrt{5}} \log |\Delta|-10}+\frac{\log \left(\pi^{-1} L\right)}{L}+\frac{C}{\frac{3}{\sqrt{5}} \log |\Delta|-10}
\end{aligned}
$$

We want to show that the right-hand side is less than 1 for large enough $|\Delta|$. Before we start, we want to give a bound on $E(\Delta)=F(\Delta)(\log |\Delta|)^{4}$. To do this, we are going to bound $\log F(\Delta)$ and $\log E(\Delta)$. By Théorème 1.1 in Rob83 we have

$$
\omega(n) \leq 1.4 \frac{\log n}{\log \log n}
$$

for any $n \geq 3$. Therefore we obtain the bound

$$
\frac{\log F(\Delta)}{\log 2} \leq 1.4 \frac{\log |\Delta|}{\log \log |\Delta|}
$$

Then the bound on $\log E(\Delta)$ is given by

$$
\log E(\Delta) \leq \frac{\log |\Delta|}{\log \log |\Delta|}+4 \log \log |\Delta| .
$$

Now we want to bound $E|\Delta|^{-1 / 2}$. Since the function

$$
u_{0}(x)=\frac{1}{\log \log x}+\frac{4 \log \log x}{\log x}-\frac{1}{2}
$$

is decreasing for $x \geq 10^{10}$ we obtain

$$
\frac{\log \left(E|\Delta|^{-1 / 2}\right)}{\log |\Delta|} \leq u_{0}\left(10^{50}\right)<-\frac{1}{10}
$$

for $|\Delta| \geq 10^{50}$. This in turn implies

$$
E|\Delta|^{-1 / 2}<|\Delta|^{-0.1} .
$$


The next step is to bound the second term of (16). The functions

$$
u_{1}(x)=\log 2 \frac{1}{\log \log x-c_{1}-\log 2}+4 \frac{\log \log x}{\log x}
$$

and

$$
u_{2}(x)=\left(\frac{3}{\sqrt{5}}-\frac{10}{\log x}\right)^{-1}
$$

are decreasing for $x \geq 10^{10}$. We have

$$
\frac{\log E}{\frac{3}{\sqrt{5}} \log |\Delta|-10} \leq u_{1}(|\Delta|) u_{2}(|\Delta|) \leq u_{1}\left(10^{50}\right) u_{2}\left(10^{50}\right) \leq 0.4896
$$

for $|\Delta| \geq 10^{50}$.

To bound the third term of 16 we remark that the function $x \mapsto x^{-1} \log \left(\pi^{-1} x\right)$ is decreasing for $x \geq e / \pi$. We have $L \geq \frac{3}{\sqrt{5}} \log |\Delta|-10 \geq e / \pi$ for $\Delta \geq 10^{15}$ and therefore

$$
\frac{\log \left(\pi^{-1} L\right)}{L} \leq \frac{\log \left(\pi^{-1}\left(\frac{3}{\sqrt{5}} \log |\Delta|-10\right)\right)}{\frac{3}{\sqrt{5}} \log |\Delta|-10} .
$$

The function

$$
u_{3}(x):=\frac{\log \left(\pi^{-1}\left(\frac{3}{\sqrt{5}} \log x-10\right)\right)}{\frac{3}{\sqrt{5}} \log x-10}
$$

is decreasing for $x \geq 10^{15}$. Thus we obtain

$$
\frac{\log \left(\pi^{-1} L\right)}{L} \leq u_{3}(|\Delta|) \leq u_{3}\left(10^{15}\right)<0.0674<\frac{1}{10}
$$

for $|\Delta| \geq 10^{50}$.

For $|\Delta| \geq e^{10 \frac{\sqrt{5}}{3}(C+1)}$ we have

$$
\frac{C}{\frac{3}{\sqrt{5}} \log |\Delta|-10} \leq \frac{1}{10} .
$$

By equation (17) we can bound the first term of (16) by

$$
\left(\frac{[\mathbb{Q}(\alpha): \mathbb{Q}] c_{2}}{2 \pi}\right)|\Delta|^{-0.1} \leq \frac{1}{10}
$$

for $|\Delta| \geq\left(10[\mathbb{Q}(\alpha): \mathbb{Q}] c_{2} /(2 \pi)\right)^{10}$.

Using these two inequalities together with $(18)$ and $(19)$ we obtain that $(16)$ is less than 1 for all

$$
|\Delta| \geq \max \left\{10^{50}, e^{10 \frac{\sqrt{5}}{3}(C+1)},\left(10[\mathbb{Q}(\alpha): \mathbb{Q}] c_{2} /(4 \pi)\right)^{10}\right\} .
$$


This contradicts the lower bound of (16).

The lower bound on $|\Delta|$ can be simplified. In equation 15$)$ we have put $C=4[\mathbb{Q}(\alpha)$ : $\mathbb{Q}] c_{2}+5 \mathcal{P}(\xi)+4 \mathcal{M}(\xi)+h(\alpha)+\log 2+0.01$. Recall that $c_{2} \geq 1$. This implies $C \geq 13$ and hence

$$
e^{15 C} \geq e^{195} \geq 10^{50}
$$

Moreover, we have

$$
\begin{aligned}
15 C \geq 10 \frac{\sqrt{5}}{3}(C+1) & \geq 10 \frac{\sqrt{5}}{3} 2[\mathbb{Q}(\alpha): \mathbb{Q}] c_{2} \geq 10 \frac{5}{4 \pi} 2[\mathbb{Q}(\alpha): \mathbb{Q}] c_{2} \\
& \geq 10 \log \left(\frac{5}{4 \pi} 2[\mathbb{Q}(\alpha): \mathbb{Q}] c_{2}\right) .
\end{aligned}
$$

Therefore, the bound on $|\Delta|$ from equation (21) simplifies to

$$
|\Delta| \geq e^{15 C},
$$

where $C>0$ is the (computable) constant

$$
C=2[\mathbb{Q}(\alpha): \mathbb{Q}] c_{2}+6 \mathcal{P}(\xi)+4 \mathcal{M}(\xi)+h(\alpha)+\log 2+0.01
$$

\section{References}

[Adl14] Semjon Adlaj: "Multiplication and division on elliptic curves, torsion points and roots of modular equations". 2014.

[BG07] Enrico Bombieri and Walter Gubler: Heights in Diophantine Geometry. Cambridge University Press, 2007.

[BHK18] Yuri Bilu, Philipp Habegger, and Lars Kühne: No singular modulus is a unit. In: International Mathematics Research Notices (2018).

[BLP16] Yuri Bilu, Florian Luca, and Amalia Pizarro-Madariaga: Rational products of singular moduli. In: Journal of Number Theory 158 (2016), pp. 397-410.

[BMZ13] Yuri Bilu, David Masser, and Umberto Zannier: "An effective "Theorem of André" for CM-points on a plane curve". In: Mathematical Proceedings of the Cambridge Philosophical Society. Vol. 154. 01. Cambridge Univ Press. 2013, pp. 145-152. 
[Coh13] Henri Cohen: A course in computational algebraic number theory. Vol. 138. Springer, 2013.

[Col98] Pierre Colmez: Sur la hauteur de Faltings des variétés abéliennes à multiplication complexe. In: Compositio Mathematica 111.3 (1998), pp. 359-369.

[Cox11] David A Cox: Primes of the form $x^{2}+n y^{2}:$ Fermat, class field theory, and complex multiplication. Vol. 34. John Wiley \& Sons, 2011.

[Dav95] Sinnou David: Minorations de formes linéaires de logarithmes elliptiques. In: Mémoires de la Societé Mathématique de France 62 (1995), pp. 1-143.

[FP87] Alain Faisant and Georges Philibert: Quelques résultats de transcendance liés à l'invariant modulaire $j$. In: Journal of number theory 25.2 (1987), pp. 184200.

[Hab15] Philipp Habegger: Singular moduli that are algebraic units. In: Algebra 8 Number Theory 9.7 (2015), pp. 1515-1524.

[Hua12] L.-K. Hua: Introduction to number theory. Springer Verlag, 2012.

[Leh42] Derrick H. Lehmer: Properties of the coefficients of the modular invariant $J(\tau)$. In: American Journal of Mathematics 64.1 (1942), pp. 488-502.

[Li18] Yingkun Li: Singular Units and Isogenies Between CM Elliptic Curves. In: arXiv preprint arXiv:1810.13214 (2018).

[Mas75] David W. Masser: Elliptic Functions and Transcendence. Vol. 437. Springer, 1975.

[NT91] Yukiyoshi Nakkajima and Yuichiro Taguchi: A generalization of the ChowlaSelberg formula. In: Journal reine angewandte Math 419 (1991), pp. 119-124.

[Rob83] Guy Robin: Estimation de la fonction de Tchebychef $\theta$ sur le k-ième nombre premier et grandes valeurs de la fonction $\omega(n)$ nombre de diviseurs premiers de n. In: Acta Arithmetica 42.4 (1983), pp. 367-389.

[Wüs14] Gisbert Wüstholz: A note on the conjectures of André-Oort and Pink. In: Bull. Ins. Math., Acad. Sinica (New Series) 9 (2014), pp. 735-779. 